\title{
Protective effects of metformin against osteoarthritis through upregulation of SIRT3-mediated PINK1/Parkin-dependent mitophagy in primary chondrocytes
}

\author{
Chenzhong Wang ${ }^{\S}$, Yi Yang ${ }^{\S}$, Yueqi Zhang, Jinyu Liu, Zhenjun Yao, Chi Zhang* \\ Department of Orthopedic surgery, Zhongshan Hospital, Fudan University, Shanghai, China.
}

\begin{abstract}
Summary Mitochondrial damage is involved in the pathogenesis of osteoarthritis. Metformin, one of the most common prescriptions for patients with type 2 diabetes, can reportedly activate Sirtuin 3 (SIRT3) expression which protects mitochondria from oxidative stress. In this study, we investigated the inhibitory property of metformin on mitochondrial damage by focusing on the interleukin-1 beta (IL-1ß)-stimulated osteoarthritis model by using primary murine chondrocytes. Our results demonstrated that SIRT3 was downregulated in chondrocytes under IL-1 $\beta$ stimulation, where its expression was positively correlated with mitochondrial damage and reactive oxygen species (ROS) production. Metformin treatment upregulated SIRT3 expression and mitigated loss of cell viability and decreased the generation of mitochondria-induced ROS in chondrocytes stimulated with IL-1ß. Metformin also attenuated IL-1 -induced expressions of catabolic genes such as matrix metalloproteinase-3 (MMP3) and MMP13 and enhanced the anabolic indicator Collagen II. These effects were mediated by phosphatase and tensin homolog (PTEN)-induced putative kinase protein 1 (PINK1)/Parkindependent mitophagy and the autophagic elimination of damaged mitochondria. Further, the SIRT3 inhibitor 3-TYP effectively inhibited the initiation of mitophagy, as decreased expression of PINK1 and Parkin, decreased the LC3II/LC3I, enhanced the expression of MMP3 and MMP13, and decreased the expression of Collagen II. Overall, our findings provide evidence that metformin suppresses IL-1 $\beta$-induced oxidative and osteoarthritis-like inflammatory changes by enhancing the SIRT3/PINK1/Parkin signaling pathway, thereby indicating metformin's potential in prevention and treatment of osteoarthritic joint disease.
\end{abstract}

Keywords: Osteoarthritis, mitophagy, SIRT3

\section{Introduction}

Osteoarthritis (OA) is a common degenerative joint disease affecting patients worldwide, which is characterized by articular cartilage degradation and elevated chondrocyte mortality, in addiction to synovial inflammation, osteophyte formation and remodeling of subchondral bone (1). It is well documented that mitochondrial dysfunction and damages can cause an

Released online in J-STAGE as advance publication December 22,2018 .

${ }^{\S}$ These authors contributed equally to this work.

*Address correspondence to:

Dr. Chi Zhang, Department of Orthopedic surgery, Zhongshan Hospital, Fudan University, 180 Fenglin Road, Shanghai 200032, China.

E-mail: zhang.chi2018@aliyun.com inflammatory response, characterized by interleukin-1 beta (IL-1 $\beta$ ) release which is a classic hallmark of OA chondrocytes (2).

The mitochondria is a membrane-enclosed organelle that converts nutritional molecules into adenosine triphosphate(ATP) via oxidative phosphorylation (OXPHOS) (3). Mitochondria are at the center of multiple specific pathways in cartilage homeostasis owing to their central role in catabolic and anabolic metabolism, generation of reactive oxygen species (ROS), apoptosis, and signal transduction. OA chondrocytes demonstrate impaired mitochondrial function (4) and decreases in mitochondrial biogenesis and OXPHOS (5).

Protein acetylation in mitochondria typically promotes decreased mitochondrial integrity and function (6). The nicotinamide adenine dinucleotide $\left(\mathrm{NAD}^{+}\right)$- 
dependent protein deacetylase, SIRT3 (Sirtuin 3), is the major mitochondrial protein deacetylase localized to the mitochondria $(7,8)$, thereby regulating mitochondrial antioxidant system and OXPHOS $(9,10)$. SIRT3 protects mitochondria from oxidative stress by deacetylating superoxide dismutase (SOD2) and mitigates OA progression (11). Chen et al. recently showed that SIRT3 was regulated by AMP-activated protein kinase (AMPK) activation in human chondrocytes, which reduced mtDNA ${ }^{4977}$ deletion and improved mitochondrial functions (12). AMPK activator metformin also increased SIRT3 expression and knockdown SIRT3 by short hairpin RNA (shRNA) transfection mediated accumulation of oxidative stress (13). In our study, we chose metformin to induce SIRT3 expression. Meanwhile considering metformin is an autophagy-inducing drug possibly through mitophagy (14), we investigated the relationship between SIRT3 and mitophagy using metformin.

The role of autophagy in human chondrocytes and the pathophysiology of OA has been elucidated, which is known to modulate OA-related gene expression and apoptosis (15). Mitophagy is the specific autophagic elimination of damaged mitochondria, regulated by the kinase phosphatase and tensin homolog (PTEN)induced putative kinase protein 1 (PINK1) and the E3 ubiquitin ligase Parkin in many metazoan cell types (16). A recent study has shown that loss of Parkin can impair the elimination of damaged/dysfunctional mitochondria in IL-1 $\beta$-stimulated OA chondrocytes (17). Together, these data suggest that the functional role of mitophagy is critical in the pathophysiology of OA.

The relationship between SIRT3 and mitophagy has been previously reported $(18,19)$ wherein depletion of SIRT3 strongly reduced mitophagy. However, to our best knowledge, the effectiveness of SIRT3-mediated mitophagy in OA has not yet been investigated. Herein, we aimed to examine the effects of SIRT3-mediated mitophagy on IL-1 $\beta$-induced mitochondrial damage and inflammatory responses in primary murine chondrocytes (PMCs).

\section{Materials and Methods}

\subsection{Materials and chemicals}

Metformin was purchased from Sigma (St.Louis, USA). It was dissolved in dimethyl sulfoxide (DMSO) for the in vitro assay and was stored at a concentration of $1 \mathrm{M}$, diluted with Dulbecco's modified Eagle's medium (DMEM), and a solvent control with DMSO was performed at no more than $2 \%$ (v/v). 3-TYP was purchased from Selleck (Shanghai, China) and was dissolved in DMSO and stored at a concentration of 5 $\mathrm{mM}$.

DMEM/F12 and fetal Bovine Serum (FBS) were from Gibco BRL (Grand Island, NY, USA). Antibodies against Parkin, LC3B and glyceraldehyde-3-phosphate dehydrogenase (GAPDH) were from Cell Signaling Technology (Boston, USA). Antibodies against PINK1, MMP3, MMP13 and Collagen IIwere from Abcam (Cambridge, UK). IL-1 $\beta$ was from Preprotech (Chicago,USA). Phenylmethylsulfonyl fluoride (PMSF), ethylenediamine tetraacetic acid (EDTA), 3-(4,5-dimethyl-2-thiazolyl)-2,5-diphenyl-2-tetrazolium bromide (MTT) and other chemicals were purchased from Sigma.

\subsection{PMC isolation and culture}

All experiments with mice were approved by the Ethics Committee for Animal Research (Zhongshan Hospital, Shanghai, China). Mouse articular chondrocytes were isolated from the knee of 6 8-week-old C57BL/6 male mice. Cartilage was dissected from the joint explant surfaces and then rinsed with saline. The cartilage explants were digested overnight at $37^{\circ} \mathrm{C}$ in $1.5 \mathrm{mg} / \mathrm{mL}$ collagenase II solution (Gibco, CA, USA). The primary isolated chondrocytes were seeded in flasks after being filtered through a $70 \mu \mathrm{M}$ cell strainer. Cells are cultured in DMEM/F12 (Gibco) with 10\% fetal bovine serum (FBS)(Gibco). Passage 2 was used for experimentation.

\subsection{Cell viability assay}

Chondrocytes were seeded in 96-well plates. After stimulation with IL-1 $\beta$ for $24 \mathrm{~h}$ in the presence or absence of different concentrations of metformin, cell viability was evaluated using the Cell Counting Kit-8 solution (Beyotime, Shanghai, China).

\subsection{Detection of reactive oxygen species (ROS)}

Intracellular ROS generation was detected by Cellular Reactive Oxygen Species Detection Assay Kit (Beyotime). Cells were incubated with $10 \mu \mathrm{M}$ 2',7'-dichlorofluorescin diacetate (DCF-DA) for $30 \mathrm{~min}$ and then washed with phosphate buffered saline (PBS) thrice. The fluorescent signals were recorded with a fluorescent microscope (Olympus, Japan). Six random fields were selected and quantified and measured by using the Image J software (National Institutes of Health, Bethesda, Maryland, USA).

\subsection{Protein isolation and western blot analysis}

To extract whole-cell proteins, the chondrocytes were collected, washed thrice with ice-cold PBS, and then lysed using the cell lysis buffer $(25 \mathrm{mM}$ tris- $\mathrm{Cl}[\mathrm{pH}$ 7.5], $250 \mathrm{mM} \mathrm{NaCl}, 5 \mathrm{mM}$ ethylenediaminetetraacetic acid, 1\%Nonidet-P40, $1 \mathrm{mM}$ phenylmethylsulphonyl fluoride, $5 \mathrm{mM}$ dithiothreitol, $2 \mathrm{X}$ protease and phosphatase inhibitor [Thermo Scientific, Waltham, MA, USA]) for $15 \mathrm{~min}$ before cell debris were removed 
by centrifugation. The same amounts of protein $(20 \mu \mathrm{g})$ were separated by sodium dodecyl sulfatepolyacrylamide gel electrophoresis (SDS-PAGE) and transferred to polyvinylidene difluoride (PVDF) membranes. The membranes were blocked with 5\% non-fat dry milk for $1 \mathrm{~h}$ at room temperature and subsequently probed with primary antibodies overnight with gentle agitation at $4{ }^{\circ} \mathrm{C}$. After washing three times with Tris-buffered saline containing $0.1 \%$ Tween-20 (TBST) for $5 \mathrm{~min}$, the membranes were incubated with the corresponding horseradishperoxidase-linked secondary antibodies (Proteintech, Wuhan, China) for $1 \mathrm{~h}$ at room temperature. The membranes were visualized by enhanced chemiluminescence (ECL) solution (Epizyme Bio, Shanghai, China) and the density of each band was measured on the Tanon Imager 4600 system (Tannon, China) and quantified by Image J software.

\subsection{Gene expression analyses by Real-time Quantitative Polymerase Chain Reaction (RT-qPCR)}

Total RNA was extracted using the TRIZOL reagent (Sigma, St. Louis, USA). cDNA was synthesized using $1 \mu \mathrm{g}$ RNA isolated from mice articular chondrocytes with the First Strand cDNA synthesis kit (Takara, Dalian, China) according to the manufacturers' recommendations. Quantitative PCR analyses were performed using SYBR Green qPCR master mix system (Takara, Dalian, China) according to the manufacturers' recommendations. The target genes expressions were normalized to the mRNA levels of the housekeeping gene GAPDH. The following conditions were used for PCR amplification: incubation for $10 \mathrm{~min}$ at $95^{\circ} \mathrm{C}$ followed by 40 amplification cycles of $15 \mathrm{~s}$ of denaturation at $95^{\circ} \mathrm{C}$ followed by $45 \mathrm{~s}$ of annealing-elongation at $60^{\circ} \mathrm{C}$. Primers used for qPCR analysis are as follows GAPDH forward primer:5'AGGTCGGTGTGAACGGATTTG-3', reverse primer: 5'-TGTAGACCATGTAGTTGAGGTCA-3'; SIRT3 forward primer: 5'-ATCCCGGACTTCAGATCCCC-3', reverse primer: 5'-CAACATGAAAAAGGGCTTGGG-3'.

\subsection{Mitochondrial specific fluorescence staining}

MitoTrackerTM Green FM (M7514, ThermoFisher) is a mitochondrial membrane potential independent mitochondrial staining regent. The experiment was performed according to the manufacturer's protocol. Mitochondria were observed under fluorescence microscopy.

\subsection{Immunofluorescence staining}

Chondrocytes were inoculated on the culture slide (Corning, Corning, NY, USA) to facilitate microscopic observation. After 4\% paraformaldehyde fixation, $0.5 \%$ Triton X-100 permeabilization and serum blocking overnight, cells were incubated with the rabbit polyclonal anti-LC3B antibodies $(1: 200)(\mathrm{CST})$ at $4^{\circ} \mathrm{C}$ overnight and then incubated with rabbit $\operatorname{IgG}(\mathrm{H}+\mathrm{L})$ secondary antibodies (Alexa Fluor 555, $1: 1000)$ (Invitrogen) at room temperature for $1 \mathrm{~h}$. The fluorescent signals were recorded with a fluorescent microscope (Olympus, Japan).

\subsection{Statistical analysis}

All experiments reported in this study were replicated in six independent experiments. The results are presented as the mean \pm SD. All data analyses were performed using GraphPad Prism 7 software (GraphPad Software Inc., San Diego, CA, USA). Statistical significance was assessed by unpaired $t$-test (between two groups) and one-way analysis of variance followed by least significant difference (LSD) test (among multiple groups). A $P$ value of $<0.05$ was considered statistically significant.

\section{Results}

\subsection{IL-1 $\beta$ reduces chondrocyte viability and metformin prevents it}

In the present study, IL-1 $\beta$ (20) significantly reduced chondrocytes viability in a dose dependent manner (Figure 1A). There was nearly a $40 \%$ decrease in cell

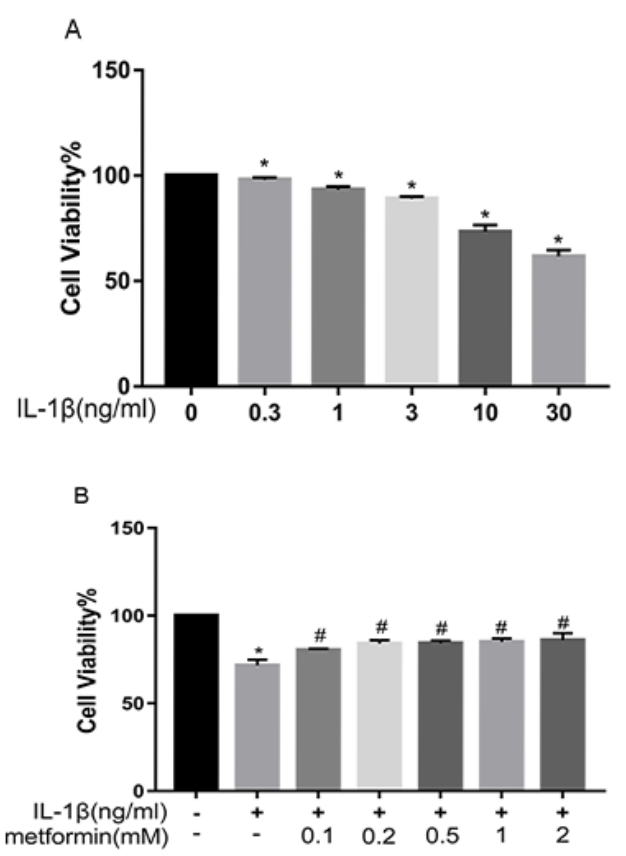

Figure 1. Effects of IL-1 $\beta$ and metformin on cell viability. (A) Cell Counting Kit-8 (CCK-8) results of primary murine articular chondrocytes treated with different concentrations of IL-1及 $(0,0.3,1,310$ and $30 \mathrm{ng} / \mathrm{mL})$ for $24 \mathrm{~h}$. (B) CCK-8 results of metformin-pretreated $(0,0.1,0.2,0.5,1$ and $2 \mathrm{mM})$ chondrocytes stimulated by $10 \mathrm{ng} / \mathrm{mL}$ IL-1 $\beta$. The data in the figures represent the averages \pm SD. Significant differences between groups are indicated as ${ }^{*} P<0.05$ compared to group $\mathrm{NC} ;{ }^{\#} P<0.05$ compared to the cells treated with IL-1 $\beta(n=6)$. 
A

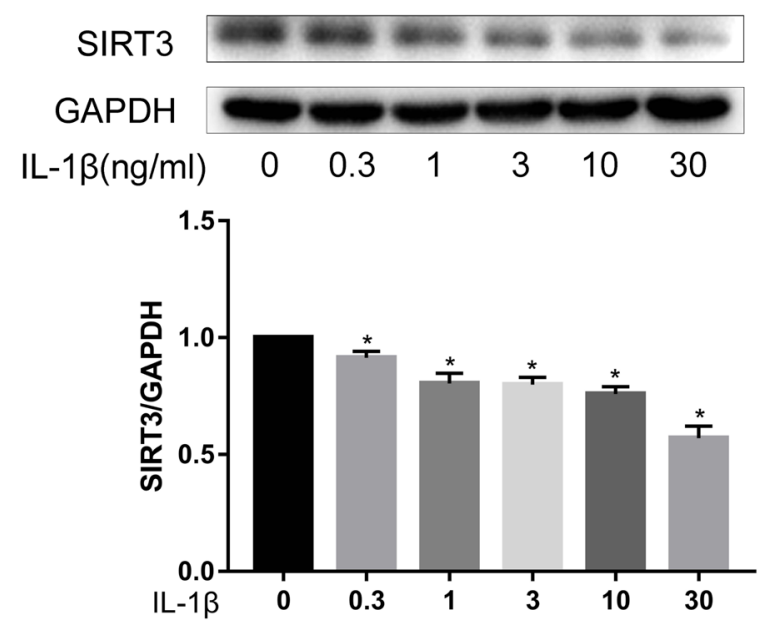

B

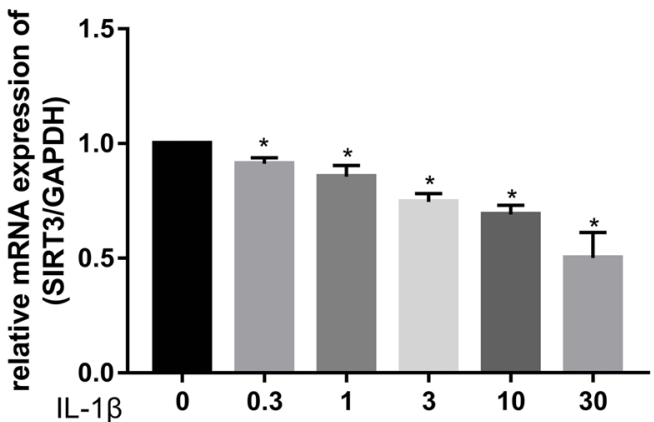

C
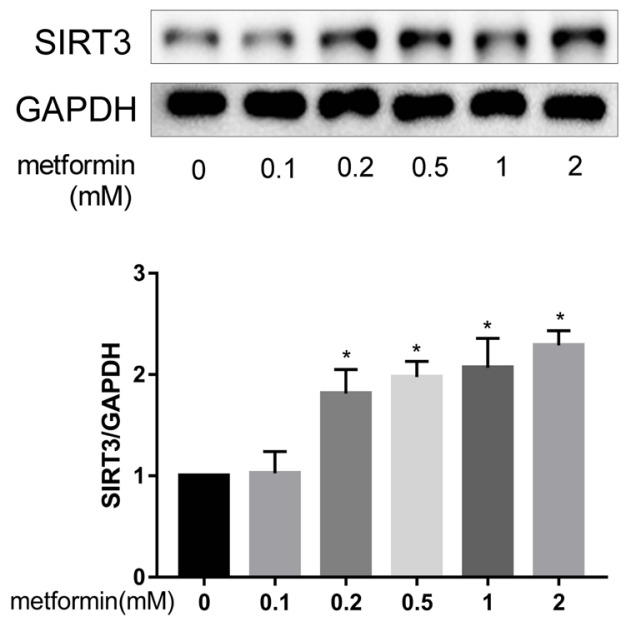

D
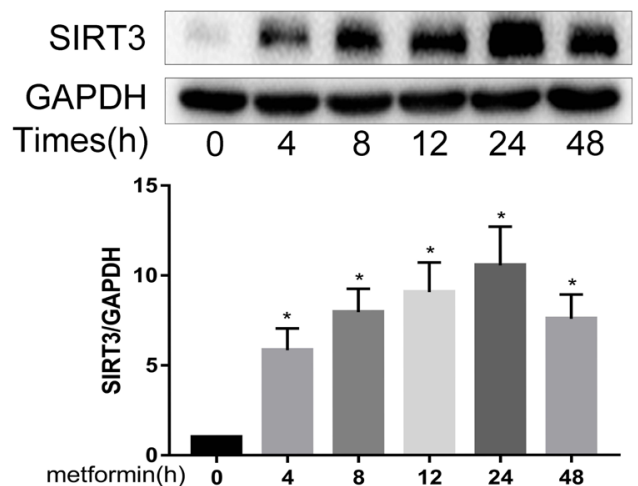

Figure 2. Effects of IL-1 $\beta$ and metformin on the expression of SIRT3. (A) Chondrocytes were incubated with IL-1 $\beta$ ( $0,0.3$, 1, 3, 10 and $30 \mathrm{ng} / \mathrm{mL}$ ) respectively, the expression of SIRT3 proteins were detected by Western blotting. (B) Chondrocytes were incubated with IL-1 $\beta(0,0.3,1,3,10$ and $30 \mathrm{ng} / \mathrm{mL})$ respectively, the level of relative mRNA expression of SIRT3 were detected by qPCR. (C) After metformin treatment $(0,0.1,0.2,0.5,1$ and $2 \mathrm{mM})$ for $24 \mathrm{~h}$, the expression of SIRT3 proteins were detected by Western blotting. (D) Chondrocytes were incubated with $1 \mathrm{mM}$ metformin for $0,4,8,12,24$ or $48 \mathrm{~h}$ respectively, the expression of SIRT3 proteins were detected by Western blotting. The data in the figures represent the averages \pm S.D. Significant differences between the treatment and control groups are indicated as ${ }^{*} p<0.05, n=6$.

viability after treatment with $30 \mathrm{ng} / \mathrm{mL}$ IL- $1 \beta$ compared with the control group $(p<0.01)$. Metformin alone did not exhibit any toxic effects on cells (data not shown). In the presence of metformin, IL- $1 \beta$-induced cell toxicity was reduced by different concentration of metformin (Figure 1B).

\subsection{SIRT3 is downregulated in chondrocytes stimulated with IL-1 , and metformin can activate SIRT3 expression}

To investigate the effect of IL-1 $\beta$ on the expression of SIRT3, the level of SIRT3 expression was detected by western blotting and qRT-PCR assays in chondrocytes stimulated with different concentration of IL- $1 \beta$. As shown in Figure 2A-B, the expression of SIRT3 decreased in a dose dependent manner. We also detected whether SIRT3 expression was regulated by metformin in chondrocytes, the cells were treated with different concentrations or with $1 \mathrm{mM}$ metformin for different time points as shown in Figure 2C-D. Metformin increased the expression of SIRT3 in a concentration- and time-dependent manner. We found that treatment with $1 \mathrm{mM}$ metformin for $24 \mathrm{~h}$ resulted in autophagy activation reaching its highest point. According to these findings, the concentration of metformin was chosen as $1 \mathrm{mM}$ and treatment course as $24 \mathrm{~h}$ for subsequent studies.

\subsection{Metformin ameliorates $I L-1 \beta$-induced oxidative stress in chondrocytes}

As oxidative stress also plays a critical role in the progression of OA (21), we performed ROS staining assay and mitochondrial membrane potential detection in chondrocytes treated by IL-1 $\beta$ and metformin to confirm whether metformin could alleviate IL-1 $\beta$ induced mitochondrial stress. As shown in Figure 3 , pretreatment with metformin could significantly decrease IL-1 $\beta$-induced ROS production and rescue downregulated mitochondrial membrane potential.

\subsection{Effect of metformin on mitochondrial function regulators in chondrocytes}




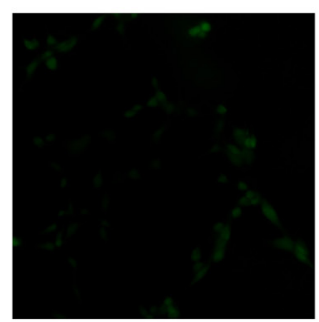

Control

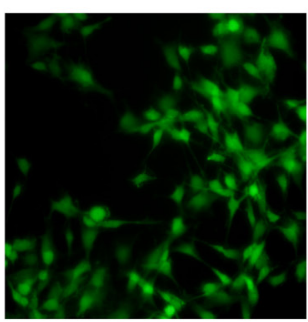

IL-1 $\beta$

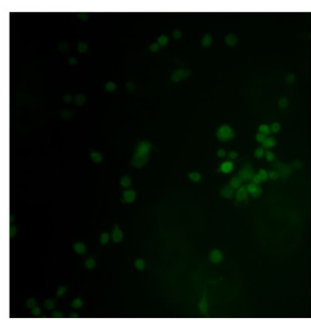

metformin+IL-1 $1 \beta$

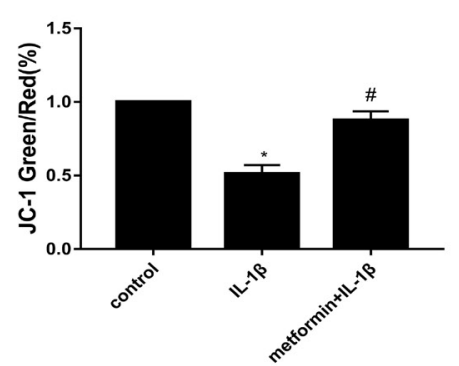

Figure 3. Metformin treatment reduces chondrocytes oxidative stress and decreases mitochondrial membrane potential. (A) ROS measurement was described as the fluorescence intensity imaged by fluorescence microscopy. (B) Mitochondrial membrane potential was described as relative mean fluorescence intensity of Green/Red. The data in the figures represent the averages \pm S.D. Significant differences between groups are indicated as $* p<0.05$ compared to group $\mathrm{NC} ; \# p<0.05$ compared to the cells treated with IL-1 $\beta(n=6)$.

A

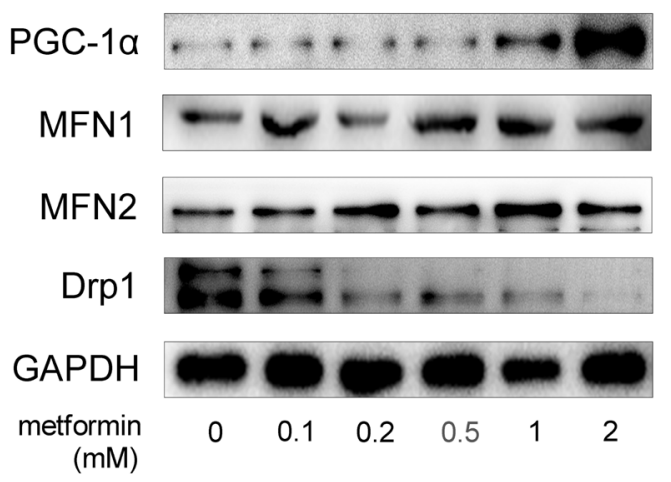

B
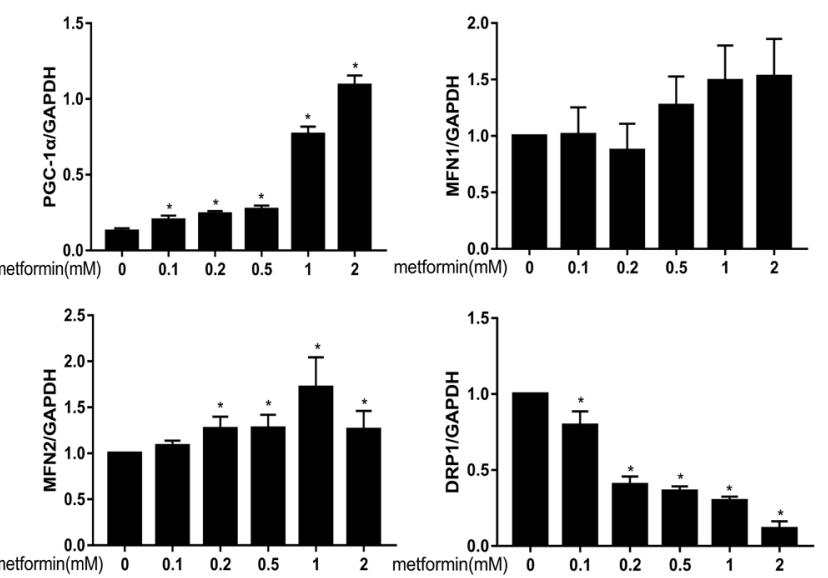

Figure 4. Effect of metformin on mitochondrial function regulators in chondrocytes. (A)The protein expression of PGC-1 $\alpha$, MFN1, MFN2, Drp1 in chondrocytes treated with different concentration of metformin $(0,0.1,0.2,0.5,1$ and $2 \mathrm{mM})$ for $24 \mathrm{~h}$. (B) Relative level of PGC-1 $\alpha$, relative level of MFN1, relative level of MFN2, relative level of Drp1. The data in the figures represent the averages \pm S.D. Significant differences between the treatment and control groups are indicated as * $p<0.05, n=6$.

To confirm the effect of metformin on mitochondrial functional statement, we screened the change of mitochondrial fission and fusion-related protein including peroxisome proliferator-activated receptor gamma coactivator 1 Alpha (PGC-1 $\alpha)$, mitofusin 1(MFN1), mitofusin 2(MFN2), dynamin-related protein 1(Drp1) (Figure 4). We found that the levels of PGC- $1 \alpha$ and MFN2 protein expression increased in a concentration-dependent manner after metformin treatment while the level of Drp1 decreased. These findings indicated metformin promoted mitochondrial fusion and inhibited mitochondrial fission which elevated mitochondrial quality.

\subsection{Metformin promotes PINK1/Parkin-mediated mitophagy in chondrocytes}

To investigate the effect of metformin on autophagy, the expression of SIRT3, PINK1, Parkin and LC3B was detected by western blotting. As shown in Figure $5 \mathrm{~A}$, the expression of SIRT3 decreased in chondrocytes stimulated with IL-1 $\beta$. Metformin upregulated the expression of SIRT3, PINK1, Parkin and the ratio of LC3II/LC3I under inflammatory cytokines stimulation.

To further confirm the effect of metformin on mitophagy, MitoTracker Green staining and LC3B immunofluorescence staining were used to detect mitophagy. Metformin significantly increased colocalization of autophagosome with mitochondria, as evidenced by the merged fluorescent signaling of LC3B and MitoTracker (Figure 6B). Taken together, these data suggest that metformin stimulates mitophagy by activating mitochondrial PINK1/Parkin signaling pathway in OA chondrocytes.

\subsection{SIRT3 inhibitor 3-TYP inhibits SIRT3-mediated PINK1/Parkin-dependent mitophagy in chondrocytes}

We further investigated the effect of SIRT3 inhibitor 3-TYP on mitophagy for the study of mechanisms involved in the SIRT3-mediated PINK1/Parkindependent mitophagy. Western blotting results showed pretreatment of 3-TYP inhibited the increase of PINK1, Parkin and the ratio of LC3II/LC3Ias compared to 
A

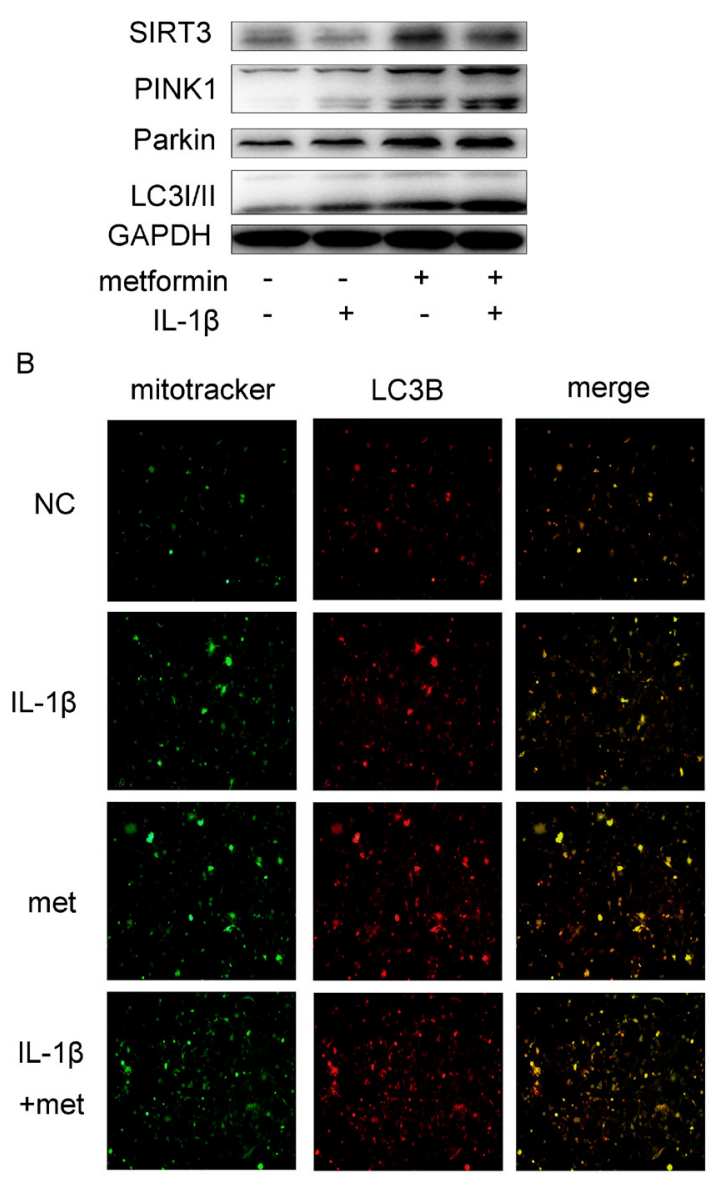

Figure 5. Metformin promotes PINK1/Parkin-mediated mitophagy in chondrocytes. (A) After $10 \mathrm{ng} / \mathrm{mL}$ IL- $1 \beta$ treatment with or without metformin for $24 \mathrm{~h}$, the expression of SIRT3, PINK1, Parkin and LC3B were determined by Western blot analysis. (B) Representative colocalization images of LC3B immunofluorescent (green) and mitochondria (MitoTracker Green). Chondrocytes were pretreated without or with IL-1 $\beta$ for $1 \mathrm{~h}$, followed by incubation with metformin for another $24 \mathrm{~h}$.

chondrocytes treated with metformin alone (Figure 6). These results indicated that SIRT3 may serve as a upstream regulator of the PINK1/Parkin/LC3B pathway.

\subsection{SIRT3/PINK1/Parkin signaling pathway is involved in the pathogenesis of $O A$}

To confirm the protective effect of SIRT3-mediated mitophagy in chondrocytes, we screened the expression of OA markers-MMP3, MMP13 and collagen II-when chondrocytes were treated with IL-1 $\beta$. As shown in Figure 7, immunoblotting revealed that inflammatory cytokine IL- $1 \beta$ increased matrix degrading enzymes, MMP3 and MMP13 and decreased collagen II, as compared to the levels in vehicle-treated chondrocytes. However, metformin reversed the inflammatory response caused by IL-1 $\beta$, which was dented by SIRT3 inhibitor 3-TYP. These findings illustrated that the inhibition of SIRT3 enhanced the chondrocyte

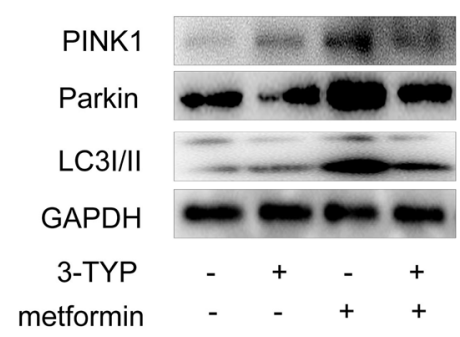

Figure 6. SIRT3 inhibitor 3-TYP inhibits SIRT3-mediated PINK1/Parkin-dependent mitophagy in chondrocytes. After $1 \mathrm{~h}$ of pretreatment without or with3-TYP $(50 \mu \mathrm{M})$, chondrocytes were treated with metformin for another $24 \mathrm{~h}$. The expression of PINK1, Parkin and LC3B was detected by Western blotting.

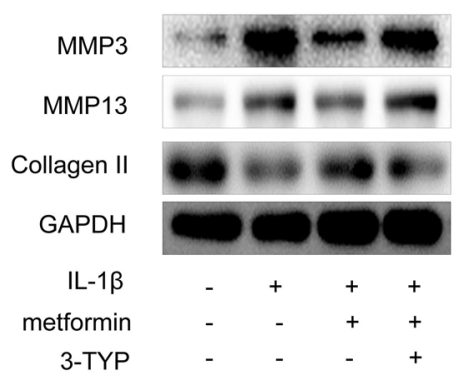

Figure 7. Effects of activation of mitophagy mediated by SIRT3 on ECM catabolism and anabolism balance. After 1 $\mathrm{h}$ of pretreatment without or with3-TYP $(50 \mu \mathrm{M})$, chondrocytes stimulated with IL-1 $\beta$ were treated with metformin for another $24 \mathrm{~h}$. The expression of MMP3, MMP13 and Collagen IIwas detected by Western blotting.

pathological phenotype via the PINK1/Parkindependent mitophagy.

\section{Discussion}

$\mathrm{OA}$ is a common disease that shows increasing incidence with age. Chondrocytes, which are the only cell type found in articular cartilages, maintain the dynamic balance between synthesis and degradation of ECM (22). Inflammatory cytokines, especially IL-1 $\beta$, were upregulated in OA joints and have been showed to decrease the production of type II collagen and increase the levels of cartilage matrix degrading proteases in chondrocytes (20). Therefore, IL-1 $\beta$ is often used to mimic pathological conditions and establish an in vitro OA model. Previous studies have shown that stimulation of primary murine chondrocytes with IL$1 \beta$ result in loss of mitochondrial membrane potential, significant increase in mitochondrial ROS levels, and chondrocyte death (17). Mitochondria dysfunction is tightly associated with oxidative stress. Therefore, we sought to establish the role of mitophagy in eliminating dysfunctional mitochondria and its impact on ROS level and chondrocyte-specific anabolic and catabolic balance under IL-1 $\beta$ stimulation in this study.

In this study, we investigated the role of SIRT3 
on mitophagy in OA. We found that SIRT3-meidated mitophagy exerted a potent protective effect against OA progression. These beneficial effects may be the result of specific autophagic elimination of damaged mitochondria.

In our study, we found that SIRT3 was decreased in IL-1 $\beta$ stimulated chondrocytes, where its expression was positively correlated with mitochondrial damage and ROS production. The results that IL- $1 \beta$ promoted generation of ROS in chondrocytes were consistent with the phenomenon that the accumulation of ROS was elevated in degenerated articular cartilage in vivo (23). We also found that metformin treatment elevated SIRT3 expression in chondrocytes. Furthermore, metformin treatment mitigated the loss of chondrocytes viability caused by IL-1 $\beta$. We also demonstrated that metformin activated SIRT3/PINK1/Parkin pathway could reverse the increased ROS level and loss of $\Delta \Psi$ M. Consistent with our results, studies have also shown that Parkin-mediated mitophagy may eliminate damaged mitochondria and decrease the level of ROS in human umbilical vein endothelial cells and fibroblasts $(24,25)$.

We also investigate the effect of metformin on mitochondrial dynamics. The dynamic balance of mitochondrial fusion and fission is critical in the maintenance of mitochondrial membrane potential and function. We found that $24 \mathrm{~h}$ of metformin treatment promoted fusion level and suppressed fission level in chondrocytes. Metformin significantly enhanced the mitochondrial fusion level and restored its normal function.

Mitophagy recently emerged as an important mechanism that controls mitochondrial quality. and as a cytoprotective mechanism to maintain mitochondrial homeostasis and cell survival under conditions of stress (26). We found that PINK1/Parkin was elevated in IL$1 \beta$ stimulated murine chondrocytes. We considered the upregulation of PINK1/Parkin as a chondrocytic response to combat pathological conditions. When metformin-mediated SIRT3 was elevated, the PINK1/Parkin pathway was more enhanced, and the immunofluorescence staining results showed enhanced mitophagy in metformin-treated and inflammationstimulated chondrocytes as compared to the IL- $1 \beta$ group, indicating that the SIRT3 regulated mitophagic activity in OA chondrocytes eventually helped to eliminate damaged mitochondria.

One of the critical events during OA is the loss of chondrocyte cellularity within the articular cartilage. This phenomenon can disrupt the balance between catabolic and anabolic processes and consequently destroy the cartilage. In our studies, metformin abolished IL-1 $\beta$-induced ECM catabolism by inducing mitophagy related to the activation of the SIRT3/ PINK1/Parkin signaling pathway. SIRT3 inhibitor 3-TYP effectively enhanced the expression of MMP3 and MMP13 and decreased the expression of collagen II. Thus, we speculate this effect of metformin on chondrocyte ECM balance may depend on the activation of PINK1/Parkin-mediated mitophagy.

In conclusion, our data reveals that SIRT3 expression is associated with the progression of OA, it is also involved in the clearance of dysfunctional mitochondria via mitophagy in chondrocytes. Metformin could suppress IL-1 $\beta$-induced oxidative stress via the activation of the SIRT3/PINK1/Parkin signaling pathway. Thus, mitophagy may become a therapeutic target for OA. Finally, owing to the association between mitophagy and OA, a wide range of drug screening targeting mitophagy may support clinical treatment of OA.

\section{Acknowledgements}

The authors thank Dr. Yi Shi, PhD, Biomedical Research Centre, Zhongshan Hospital, Fudan University, for technical assistance.

Author contributions: All listed authors have made substantial contributions to the following aspects of the manuscript: $i$ ) The conception and design of the study, or acquisition of data, or analysis and interpretation of data. ii) Drafting the article or revising it critically for important intellectual content. iii) Final approval of the version to be submitted.

Conflict of interest statement: None of the authors had financial or personal relationships with people or organizations that could inappropriately influence the bias of the presented work.

\section{References}

1. Glyn-Jones S, Palmer AJ, Agricola R, Price AJ, Vincent TL, Weinans H, Carr AJ. Osteoarthritis. Lancet. 2015; 386:376-387.

2. Blanco FJ, Rego I, Ruiz-Romero C. The role of mitochondria in osteoarthritis. Nat Rev Rheumatol. 2011; 7:161-169

3. Henze K, Martin W. Evolutionary biology: essence of mitochondria. Nature.2003; 426:127-128.

4. Maneiro E, Martín MA, Andres MCD, LópezArmada MJ, Hoyo PD, Galdo F, Arenas J, Blanco FJ. Mitochondrial respiratory activity is altered in osteoarthritic human articular chondrocytes. Arthritis Rheum. 2003; 48:700-708.

5. Wang Y, Zhao X, Lotz M, Terkeltaub R, Liubryan R. Mitochondrial biogenesis is impaired in osteoarthritis chondrocytes but reversible via peroxisome proliferatoractivated receptor $\gamma$ coactivator $1 \alpha$. Arthritis Rheumatol. 2015; 67:2141-2153.

6. Baeza J, Smallegan MJ, Denu JM. Mechanisms and dynamics of protein acetylation in mitochondria. Trends Biochem Sci. 2016; 41:231-244.

7. Onyango P, Celic I, Mccaffery JM, Boeke JD, Feinberg 
AP. SIRT3, a human SIR2 homologue, is an NADdependent deacetylase localized to mitochondria. Proc Natl Acad Sci U S A. 2002; 99:13653-13658.

8. Lombard DB, Alt FW, Cheng HL, Bunkenborg J, Streeper RS, Mostoslavsky R, Kim J, Yancopoulos G, Valenzuela D, Murphy AJM, Biology C. Mammalian Sir2 homolog SIRT3 regulates global mitochondrial lysine acetylation. Mol Cell Biol.2007; 27:8807-8814.

9. Kincaid B, Bossywetzel E. Forever young: SIRT3 a shield against mitochondrial meltdown, aging, and neurodegeneration. Front Aging Neurosci. 2013; 5:48.

10. Ansari A, Rahman MS, Saha SK, Saikot FK, Deep A, Kim KH. Function of the SIRT3 mitochondrial deacetylase in cellular physiology, cancer, and neurodegenerative disease. Aging Cell. 2017; 16:4-16.

11. Fu Y, Kinter M, Hudson J, Humphries KM, Lane RS, White JR, Hakim M, Pan Y, Verdin E, Griffin TM. Aging promotes sirtuin 3-dependent cartilage superoxide dismutase 2 acetylation and osteoarthritis. Arthritis Rheumatol. 2016; 68:1887-1898.

12. Chen LY, Wang Y, Terkeltaub R, Liu-Bryan R. Activation of AMPK-SIRT3 signaling is chondroprotective by preserving mitochondrial DNA integrity and function. Osteoarthritis Cartilage. 2018; 26:1539-1550.

13. Karnewar S, Neeli PK, Panuganti D, Kotagiri S, Mallappa S, Jain N, Jerald MK, Kotamraju S. Metformin regulates mitochondrial biogenesis and senescence through AMPK mediated H3K79 methylation: Relevance in ageassociated vascular dysfunction. Biochim Biophys Acta Mol Basis Dis. 2018; 1864:1115-1128.

14. Kim J, Yang G, Kim Y, Kim J, Ha J. AMPK activators: mechanisms of action and physiological activities. Exp Mol Med. 2016; 48:e224

15. Sasaki H, Takayama K, Matsushita T, Ishida K, Kubo S, Matsumoto T, Fujita N, Oka S, Kurosaka M, Kuroda R. Autophagy modulates osteoarthritis-related gene expression in human chondrocytes. Arthritis Rheum. 2012; 64:1920-1928.

16. Youle RJ, Narendra DP. Mechanisms of mitophagy. Nat Rev Mol Cell Biol. 2011; 12:9-14.

17. Ansari MY, Khan NM, Ahmad I, Haqqi TM. Parkin clearance of dysfunctional mitochondria regulates ROS levels and increases survival of human chondrocytes. Osteoarthritis Cartilage. 2017; 26:1087-1097.

18. Yu W, Gao B, Na L, Wang J, Qiu C, Zhang G, Min L, Zhang R, Li C, Gang J. Sirt3 deficiency exacerbates diabetic cardiac dysfunction: Role of Foxo3A-Parkinmediated mitophagy. Biochim Biophys Acta Mol Basis Dis. 2016; 1863:1973-1983.

19. Feng J, Lu C, Dai Q, Sheng J, Xu M. Pharmacology. SIRT3 facilitates amniotic fluid stem cells to repair diabetic nephropathy through protecting mitochondrial homeostasis by modulation of mitophagy. Cell Physiol Biochem. 2018; 46:1508-1524.

20. Goldring MB, Birkhead JR, Suen LF, Yamin R, Mizuno S, Glowacki J, Arbiser JL, Apperley JF. Interleukin-1 beta-modulated gene expression in immortalized human chondrocytes. J Clin Invest. 1994; 94:2307-2316.

21. Lepetsos P, Papavassiliou AG. ROS/oxidative stress signaling in osteoarthritis. Biochim Biophys Acta. 2016; 1862: 576-591.

22. Zhang Y, Vasheghani F, Li YH, Blati M, Simeone K, Fahmi H, Lussier B, Roughley P, Lagares D, Pelletier JP, Martel-Pelletier J, Kapoor M. Cartilage-specific deletion of mTOR upregulates autophagy and protects mice from osteoarthritis. Ann Rheum Dis. 2015; 74:1432-1440.

23. Jallali N, Ridha H, Thrasivoulou C, Underwood C, Butler PE, Cowen T. Vulnerability to ROS-induced cell death in ageing articular cartilage: the role of antioxidant enzyme activity. Osteoarthritis Cartilage. 2005; 13:614-622.

24. Rojansky R, Cha MY, Chan DC. Elimination of paternal mitochondria in mouse embryos occurs through autophagic degradation dependent on PARKIN and MUL1. eLife. 2016; 5:pii:e17896.

25. Zhu W, Yuan Y, Liao G, Li L, Liu J, Chen Y, Zhang J, Cheng J, Lu Y, Mesenchymal stem cells ameliorate hyperglycemia-induced endothelial injury through modulation of mitophagy. Cell Death Dis. 2018; 9:837.

26. Eiyama A, Okamoto K. PINK1/Parkin-mediated mitophagy in mammalian cells. Curr Opin Cell Biol. 2015; 33:95-101.

(Received October 31, 2018; Revised December 14, 2018; Accepted December 16, 2018) 